\title{
On the sequence of numbers of the form
}$$
\varepsilon_{0}+\varepsilon_{1} q+\ldots+\varepsilon_{n} q^{n}, \varepsilon_{i} \in\{0,1\}
$$ \\ by \\ PAul Erdős (Budapest), István Joó (Budapest) and \\ Vilmos KomorniK (Strasbourg)
}

1. Introduction. Fix a real number $1<q<2$. For every nonnegative integer $k$ let

$$
k=\varepsilon_{0}+2 \varepsilon_{1}+\ldots+2^{n} \varepsilon_{n}, \quad \varepsilon_{i} \in\{0,1\}
$$

be its dyadic expansion and set

$$
x_{k}=\varepsilon_{0}+\varepsilon_{1} q+\ldots+\varepsilon_{n} q^{n} .
$$

Denote by $y_{0}<y_{1}<\ldots$ the increasing rearrangement of the sequence $\left(x_{k}\right)$, without repetitions. It is clear that

and that

$$
y_{0}=0, \quad y_{1}=1, \quad y_{2}=q
$$

$$
y_{k} \rightarrow \infty \quad \text { if } k \rightarrow \infty .
$$

We are interested here in the behavior of the difference sequence $y_{k+1}-$ $y_{k}$. Let us introduce for brevity the following notations:

$$
l(q)=\inf \left(y_{k+1}-y_{k}\right), \quad L(q)=\lim \sup \left(y_{k+1}-y_{k}\right) .
$$

Note that $l(q)=\liminf \left(y_{k+1}-y_{k}\right)$. Indeed, fix $\varepsilon>0$ arbitrarily. It is sufficient to show that there exist arbitrarily large integers $m<l$ such that $y_{l}-y_{m}<l(q)+\varepsilon$. By the definition of $l(q)$ there exists an integer $k$ such that $y_{k+1}-y_{k}<l(q)+\varepsilon$. Then for every sufficiently large integer $n$ (such that $\left.q^{n}>y_{k+1}\right)$ the numbers $q^{n}+y_{k}$ and $q^{n}+y_{k+1}$ are in the sequence $\left(y_{i}\right)$. Denoting them by $y_{m}$ and $y_{l}$ we have $y_{l}-y_{m}=y_{k+1}-y_{k}<l(q)+\varepsilon$ and $l, m \rightarrow \infty$ as $n \rightarrow \infty$. Hence the claim follows.

We recall the following results; the first three of them were proved in [3], while the last one was obtained in [2].

1991 Mathematics Subject Classification: Primary 11B83; Secondary 11R06.

The second author was supported by the Hungarian Scientific Research Grant OTKA T 014244 . 
(a) $0 \leq l(q) \leq L(q) \leq 1$ for all $1<q<2$.

(b) $L(q)=1$ for all $A \leq q<2$ where $A=(1+\sqrt{5}) / 2$.

(c) $L(q)>0$ for all Pisot numbers.

(d) $l(q)=1 / q>0$ for all Pisot numbers $1<q<2$ satisfying the equation $q^{r+1}=1+q+\ldots+q^{r}$ for some integer $r \geq 1$.

In the proof of (b) it was assumed that $q>A$, but the proof remains valid for $q=A$. (One can also give a different proof by adapting that of Proposition 3 below; see the remark following that proposition.)

In this paper we obtain several new estimates of $l(q)$ and of $L(q)$ for some special classes of numbers $1<q<2$. In particular, we obtain the following two results:

(e) $l(q)>0$ for all Pisot numbers.

(f) $L(q)=0$ (i.e. $y_{k+1}-y_{k} \rightarrow 0$ ) for all transcendental numbers $1<q$ $<\sqrt{2}$.

The property (e) was also obtained independently in another way by $\mathrm{Y}$. Bugeaud [1]. He also proved a partial converse of this statement.

At the end of the paper we correct a small error in our previous paper [3] and we formulate some open problems.

The authors are grateful to the referee for his suggestions.

2. Pisot numbers. For any real number $x$ let us denote by $\|x\|$ its distance from the closest integer. Our main result is the following:

Theorem 1. We have $l(q)>0$ for all Pisot numbers. More precisely,

$$
L(q) \geq q^{-N}\left(1-\sum_{k=N}^{\infty}\left\|q^{k}\right\|\right)
$$

for all nonnegative integers $N$, and

$$
l(q) \geq q^{-N}\left(1-\sum_{k=N}^{\infty}\left\|q^{k}\right\|\right)
$$

for all nonnegative integers $N$ satisfying

$$
\sum_{k=N}^{\infty}\left\|q^{k}\right\|<\frac{1}{q+1} .
$$

Proof. Since $\left\|q^{k}\right\| \rightarrow 0$ exponentially if $q$ is a Pisot number, the inequality (5) is satisfied if $N$ is sufficiently large. Then it follows from (4) and (5) that

$$
l(q) \geq q^{1-N} /(q+1)>0 .
$$

It remains to prove the estimates (3) and (4). 
For every real $x$ there is a unique integer $m$ satisfying $-1 / 2<x-m \leq$ $1 / 2$. Set $d(x)=x-m$. Then $-1 / 2<d(x) \leq 1 / 2$ and $|d(x)|=\|x\|$.

There is nothing to prove if $\sum_{k=N}^{\infty}\left\|q^{k}\right\| \geq 1$. Fix a nonnegative integer $N$ such that

$$
\sum_{k=N}^{\infty}\left\|q^{k}\right\|<1
$$

and set

$$
\alpha=-\sum_{k=N}^{\infty} \min \left\{d\left(q^{k}\right), 0\right\}, \quad \beta=\sum_{k=N}^{\infty} \max \left\{d\left(q^{k}\right), 0\right\} .
$$

Then

$$
\alpha \geq 0, \quad \beta \geq 0 \quad \text { and } \quad \alpha+\beta=\sum_{k=N}^{\infty}\left\|q^{k}\right\|<1 .
$$

Consider the increasing sequence $y_{0}^{N}<y_{1}^{N}<\ldots$ of the numbers of the form (2) with $\varepsilon_{0}=\ldots=\varepsilon_{N-1}=0$. Since clearly $y_{k}^{N}=q^{N} y_{k}$ for all $k \geq 0$, it is sufficient to prove that

$$
y_{k+1}^{N}-y_{k}^{N} \geq 1-\sum_{j=N}^{\infty}\left\|q^{j}\right\|
$$

for infinitely many $k \geq 0$, and that

$$
y_{k+1}^{N}-y_{k}^{N} \geq 1-\sum_{j=N}^{\infty}\left\|q^{j}\right\|
$$

for all $k \geq 0$ if the condition (5) is satisfied.

It follows from (6) and (7) that for every $k \geq 0$ there is a unique integer $m=m(k)$ satisfying $m-\alpha \leq y_{k}^{N} \leq m+\beta$. Since $y_{k}^{N} \rightarrow \infty$, there are infinitely many $k$ 's for which $m(k)<m(k+1)$. For these $k$ 's we have (writing $m=m(k)$ )

$$
y_{k+1}^{N}-y_{k}^{N} \geq(m+1-\alpha)-(m+\beta)=1-(\alpha+\beta)=1-\sum_{j=N}^{\infty}\left\|q^{j}\right\|
$$

and (8) follows.

Now assume (5). It follows from (6) and (7) that for any $l>k \geq 0$ we have either $0<y_{l}^{N}-y_{k}^{N} \leq \alpha+\beta$ or $y_{l}^{N}-y_{k}^{N} \geq 1-(\alpha+\beta)$. It remains to prove that the first case never occurs.

Assume on the contrary that $0<y_{l}^{N}-y_{k}^{N} \leq \alpha+\beta$ for some $l>k \geq 0$. Choose an integer $m \geq 1$ such that $\alpha+\beta<q^{m}\left(y_{l}^{N}-y_{k}^{N}\right) \leq q(\alpha+\beta)$ and consider the numbers $y_{l^{\prime}}^{N}=q^{m} y_{l}^{N}$ and $y_{k^{\prime}}^{N}=q^{m} y_{k}^{N}$. Then $\alpha+\beta<y_{l^{\prime}}^{N}-y_{k^{\prime}}^{N} \leq$ $q(\alpha+\beta)$. However, this is impossible because $q(\alpha+\beta)<1-(\alpha+\beta)$ by the assumption (5). 
EXAmPLeS. 1 . Let $q \approx 1.32472$ be the first Pisot number (the real root of $\left.q^{3}-q-1=0\right)$. Denoting its conjugates by $q_{2}$ and $q_{3}$, we have the (crude) estimates $\left\|q^{k}\right\| \leq\left|q_{2}\right|^{k}+\left|q_{3}\right|^{k}$ for all $k \geq 0$. Applying the theorem with $N=22$ resp. $N=26$ and using these estimates we easily obtain $L(q)>0.0006$ and $l(q)>0.0004$.

2 . Let $q \approx 1.46557$ be the fourth Pisot number (the real root of $q^{3}-$ $\left.q^{2}-1=0\right)$. Applying the theorem with $N=15$ and with $N=18$ we easily obtain $L(q)>0.0011$ and $l(q)>0.0006$. Simple numerical tests seem to indicate that $L(q)=q-1 \approx 0.46557$ and $l(q)=q^{5}-q^{4}-q^{3}+q^{2}-1 \approx 0.1479$.

3. Let $q=A=(1+\sqrt{5}) / 2$. Applying the theorem with $N=4$ we find that $l(q)>0.09$. We recall from [2] (see (d) in the introduction) that $l(A)=1 / A \approx 0.618$. We also recall that $L(A)=1$.

We can give lower bounds of $L(q)$ and $l(q)$ without using $N$.

Corollary 2. Let $q$ be a Pisot number. Denote by $d$ the degree of its minimal polynomial, by $q_{2}, \ldots, q_{d}$ the conjugates of $q$ and by $Q$ the largest absolute value of these conjugates, so that $Q<1$. Then

$$
L(q) \geq(2 q)^{-1} q^{(\log (2 d-2)-\log (1-Q)) / \log Q}
$$

and

$$
l(q) \geq(1+q)^{-1} q^{(\log (d-1)+\log (1+q)-\log (1-Q)) / \log Q} .
$$

Proof. If we choose $N$ such that

$$
\sum_{k=N}^{\infty}\left\|q^{k}\right\|<0.5
$$

then $L(q)>2^{-1} q^{-N}$ by the preceding theorem. Since

$$
\sum_{k=N}^{\infty}\left\|q^{k}\right\| \leq \sum_{k=N}^{\infty} \sum_{j=2}^{d}\left|q_{j}\right|^{k} \leq(d-1) \sum_{k=N}^{\infty} Q^{k}=(d-1) Q^{N} /(1-Q),
$$

it is sufficient to choose $N$ so that $(d-1) Q^{N} /(1-Q)<0.5$, or equivalently,

$$
N>\frac{\log (1-Q)-\log (d-1)-\log 2}{\log Q} .
$$

Choosing the smallest integer $N$ satisfying this inequality, we have

$$
N-1 \leq \frac{\log (1-Q)-\log (d-1)-\log 2}{\log Q}
$$

and therefore

$$
L(q)>2^{-1} q^{-N}=(2 q)^{-1} q^{-(N-1)} \geq(2 q)^{-1} q^{(\log (d-1)+\log 2-\log (1-Q)) / \log Q},
$$

proving the first part of the corollary. 
Next, if we choose $N$ such that

$$
\sum_{k=N}^{\infty}\left\|q^{k}\right\|<1 /(1+q),
$$

then $l(q)>q^{1-N}(1+q)^{-1}$. By (11) it is sufficient to choose $N$ so that

$$
(d-1) Q^{N} /(1-Q)<1 /(1+q),
$$

or equivalently,

$$
N>\frac{\log (1-Q)-\log (d-1)-\log (1+q)}{\log Q} .
$$

Choosing the smallest integer $N$ satisfying this inequality, we have

$$
N-1 \leq \frac{\log (1-Q)-\log (d-1)-\log (1+q)}{\log Q}
$$

and therefore

$$
l(q)>q^{1-N}(1+q)^{-1} \geq(1+q)^{-1} q^{(\log (d-1)+\log (1+q)-\log (1-Q)) / \log Q},
$$

proving the second part of the corollary.

It is possible to obtain more accurate lower bounds of $L(q)$ by ad hoc arguments for special Pisot numbers. Let us give an example.

Proposition 3. If $q \approx 1.46557$ is the fourth Pisot number (i.e. the only real root of the equation $\left.q^{3}=q^{2}+1\right)$, then none of the open intervals $\left(q^{n}-(q-1), q^{n}\right)$ contains any element $y_{k}$. Hence $L(q) \geq q-1$.

Proof. Assume that this is false and let $n \geq 0$ be the smallest integer such that there exists $y_{k} \in\left(q^{n}-(q-1), q^{n}\right)$. It follows easily from the relations

$$
y_{0}=0, \quad y_{1}=1, \quad y_{2}=q, \quad y_{3}=q^{2}, \quad y_{4}=q+1
$$

that $n \geq 4$. Furthermore, we have obviously

$$
y_{k}=\varepsilon_{0}+\varepsilon_{1} q+\ldots+\varepsilon_{n-1} q^{n-1} .
$$

Observe that $\varepsilon_{n-1}=0$. Indeed, otherwise we would have

$$
y_{l}:=y_{k}-q^{n-1} \in\left(q^{n-3}-(q-1), q^{n-3}\right),
$$

contradicting the minimality of $n$.

Similarly, we have $\varepsilon_{n-3}=0$, for otherwise

$$
y_{l}:=y_{k}-q^{n-3} \in\left(q^{n-1}-(q-1), q^{n-1}\right),
$$

again contradicting the minimality of $n$.

Next we claim that $\varepsilon_{n-2}=1$. Indeed, otherwise $y_{k}$ would be too small: we would have $y_{k} \leq q^{n}-(q-1)$ by the following computation: 


$$
\begin{aligned}
q^{n}-(q-1)-y_{k} & \geq q^{n}-(q-1)-\left(1+q+\ldots+q^{n-4}\right) \\
& =q^{n}-q+1-\frac{q^{n-3}-1}{q-1} \\
& =\frac{q^{n+1}-q^{n}-q^{2}+2 q-1-q^{n-3}+1}{q-1} \\
& =\frac{q^{n-2}-q^{2}+2 q-q^{n-3}}{q-1}=\frac{q^{n-3}(q-1)+q(2-q)}{q-1}>0 .
\end{aligned}
$$

Now it follows that $\varepsilon_{n-4}=0$. Indeed, otherwise

$$
y_{l}:=y_{k}-q^{n-2}-q^{n-4}=y_{k}-q^{n-1} \in\left(q^{n-3}-(q-1), q^{n-3}\right),
$$

contradicting the minimality of $n$.

However, this is also impossible, because now we have $y_{k}<q^{n}-(q-1)$. Indeed, using also the relation $q^{2}(q-1)=1$ and the inequality $q>\sqrt{2}$, we obtain

$$
\begin{aligned}
q^{n}-y_{k} & \geq q^{n}-\left(1+q+\ldots+q^{n-5}+q^{n-2}\right) \\
& =q^{n}-q^{n-2}-\frac{q^{n-4}-1}{q-1}=\frac{q^{n}(q-1)-q^{n-2}(q-1)-q^{n-4}+1}{q-1} \\
& =\frac{q^{n-2}-2 q^{n-4}+1}{q-1}>\frac{1}{q-1} .
\end{aligned}
$$

REMARK. One can prove by a similar but simpler argument that if $q=A$, then none of the open intervals $\left(q^{n}, q^{n}+1\right)(n=1,2, \ldots)$ contains any element of the sequence $\left(y_{k}\right)$. Hence $L(q)=1$.

3. Numbers $q$ close to 1 . We do not know whether $L(q)=0$ for all $q$ sufficiently close to 1 . We have the following weaker result:

Theorem 4. We have $L(q) \rightarrow 0$ as $q \rightarrow 1$. More precisely, $L(q) \leq$ $\left(q^{2}-1\right)$ e for all $1<q<2$.

Proof. If $q \geq 6 / 5$, then $\left(q^{2}-1\right) e>1$ and the estimate follows from the inequality $L(q) \leq 1$. Assume therefore that $1<q<1.2$; then there exists an odd integer $n \geq 5$ satisfying

$$
1+\frac{1}{n+2} \leq q<1+\frac{1}{n}
$$

Consider the numbers $q<q^{3}<\ldots<q^{n+2}$. First of all, we have

$$
q^{n+2} \geq\left(\frac{n+3}{n+2}\right)^{n+2}>\left(\frac{4}{3}\right)^{3}>2+\frac{1}{3}>q+1
$$

because $n \geq 3$. Furthermore,

$$
q^{3}-q<q^{5}-q^{3}<\ldots<q^{n+2}-q^{n}
$$


and

$$
q^{n+2}-q^{n}=\left(q^{2}-1\right) q^{n}<\left(q^{2}-1\right) e=: \delta .
$$

We claim that for every real number $\alpha>q$ there exists a $y_{k}$ satisfying $\alpha-\delta<y_{k}<\alpha$. Indeed, since $1<q^{2}<2$, we have $L\left(q^{2}\right) \leq 1$ by (a) of the introduction. Hence there exists

$$
\bar{y}=\varepsilon_{0}+\varepsilon_{2} q^{2}+\ldots+\varepsilon_{2 m} q^{2 m}
$$

such that $\alpha-q-1 \leq \bar{y}<\alpha-q$. Consider the numbers

$$
\bar{y}+q<\bar{y}+q^{3}<\ldots<\bar{y}+q^{n+2} .
$$

The first of them is clearly less than $\alpha$, while the last one is greater than $\alpha$ :

$$
\bar{y}+q^{n+2}>\bar{y}+q+1 \geq \alpha .
$$

Furthermore, the distance of two consecutive numbers is always less than $\delta$. It follows that if we denote by $y_{k}$ the largest term of this sequence which is still less than $\alpha$, then $\alpha-\delta<y_{k}<\alpha$.

The above claim implies that

$$
\lim \sup \left(y_{k+1}-y_{k}\right) \leq \delta
$$

and the proof is complete.

Our next result shows that $y_{k+1}-y_{k} \rightarrow 0$ for almost all numbers $q$ sufficiently close to 1 .

THEOREM 5. Let $q$ be a real number satisfying $1<q<\sqrt{2}$ and $l\left(q^{2}\right)=0$. Then $L(q)=0$, i.e. $y_{k+1}-y_{k} \rightarrow 0$. In particular, this is true when $1<q<\sqrt{2}$ and $q$ is transcendental.

We need three lemmas.

Lemma 6. Let $1<q<2$ satisfy $l(q)=0$ and fix $\delta>0$. Then there exists a subsequence $\left(z_{k}\right)$ of $\left(y_{k}\right)$ satisfying the following two conditions:

(a) if $i \neq j$, then $z_{i}$ and $z_{j}$ have no common term $q^{n}$;

(b) $\delta<z_{2 i}-z_{2 i-1}<2 \delta$ for all $i=1,2, \ldots$

Proof. Since $l(q)=0$, there exist $l>k \geq 1$ such that $0<y_{l}-y_{k}<\delta$. (We may even choose $l=k+1$.) By omitting the common terms $q^{n}$ (if any), we may assume that $y_{k}$ and $y_{l}$ have no common terms. Choose a positive integer $m$ such that $\delta<q^{m}\left(y_{l}-y_{k}\right)<2 \delta$ (possible because $1<q<2$ ), and set $z_{1}=q^{m} y_{k}, z_{2}=q^{m} y_{l}$.

Now we proceed by induction. Assume that $z_{1}<\ldots<z_{2 n}$ are already defined for some $n \geq 1$ and that they satisfy the conditions (a) and (b).

Fix a positive integer $N$ such that $q^{N}>z_{2 n}$. Then none of the numbers $z_{1}<\ldots<z_{2 n}$ contains any term $q^{i}$ with $i \geq N$. Since $l(q)=0$, there exist $l>k \geq 1$ such that $0<y_{l}-y_{k}<q^{-N} \delta$. We may also assume that $y_{k}$ and $y_{l}$ have no common terms. Choose a positive integer $m$ such that 
$q^{-N} \delta<q^{m}\left(y_{l}-y_{k}\right)<2 q^{-N} \delta$ (possible because $1<q<2$ ), and set $z_{2 n+1}=$ $q^{N+m} y_{k}, z_{2 n+2}=q^{N+m} y_{l}$. Then $\delta<z_{2 n+2}-z_{2 n+1}<2 \delta$. Furthermore, $z_{2 n+2}, z_{2 n+1}$ have no common term, and no term $q^{i}$ with $i \leq N$. Hence the properties (a) and (b) continue to hold.

Lemma 7. Let $1<q<2$ satisfy $l(q)=0$ and fix $\delta>0, D>0$. Then there exists a finite subsequence

$$
w_{0}<w_{1}<\ldots<w_{m}
$$

of $\left(y_{k}\right)$ such that

$$
w_{i}-w_{i-1}<2 \delta, \quad i=1, \ldots, m
$$

and

$$
w_{m}-w_{0}>D .
$$

Pro of. Consider the sequence $\left(z_{k}\right)$ of the preceding lemma. Choose an integer $m>D / \delta$ and define

$$
\begin{gathered}
w_{0}=z_{1}+z_{3}+z_{5}+\ldots+z_{2 m-3}+z_{2 m-1}, \\
w_{1}=z_{2}+z_{3}+z_{5}+\ldots+z_{2 m-3}+z_{2 m-1}, \\
w_{2}=z_{2}+z_{4}+z_{5}+\ldots+z_{2 m-3}+z_{2 m-1}, \\
\vdots \\
w_{m-1}=z_{2}+z_{4}+z_{6}+\ldots+z_{2 m-2}+z_{2 m-1}, \\
w_{m}=z_{2}+z_{4}+z_{6}+\ldots+z_{2 m-2}+z_{2 m} .
\end{gathered}
$$

We clearly have (12) and it follows from property (a) of the preceding lemma that $\left(w_{i}\right)$ is a subsequence of $\left(y_{k}\right)$. It is also clear from (b) that (13) is satisfied. Finally, (14) also follows from (b):

$$
w_{m}-w_{0}=\left(z_{2}-z_{1}\right)+\ldots+\left(z_{2 m}-z_{2 m-1}\right)>m \delta>D .
$$

Lemma 8. If $1<q<2$ and $q$ does not satisfy any algebraic equation with integer coefficients belonging to the set $\{-1,0,1\}$, then $l(q)=0$.

Proof. Fix $\delta>0$. Choose a sufficiently large $n$ with $\left(q^{n}-1\right) /(q-1)<$ $\left(2^{n}-1\right) \delta$ and consider the numbers $x_{i}, 0 \leq i<2^{n}$, constructed in the introduction. It follows from our assumption on $q$ that they are all different. Furthermore, all these $2^{n}$ numbers belong to the interval $\left[0,1+\ldots+q^{n-1}\right]$ whose length is less than $\left(2^{n}-1\right) \delta$ by the choice of $n$. Therefore, by the box principle there are two $x_{i}$ whose distance is less than $\delta$. Hence $l(q)<\delta$. Letting $\delta \rightarrow 0$ we conclude that $l(q)=0$.

Proof of Theorem 5. Fix $\delta>0$ and apply Lemma 7 with $q^{2}$ instead of $q$. It follows that there exists a finite sequence $a_{0}<a_{1}<\ldots<a_{m}$ of numbers 
of the form

$$
\varepsilon_{0}+\varepsilon_{2} q^{2}+\varepsilon_{4} q^{4}+\ldots+\varepsilon_{2 n} q^{2 n}, \quad \varepsilon_{i} \in\{0,1\}
$$

satisfying

$$
0<a_{i}-a_{i-1}<2 \delta, \quad i=1, \ldots, m, \quad a_{m}-a_{0}>q .
$$

On the other hand, since $L\left(q^{2}\right) \leq 1$ (see (a) in the introduction), every open interval $I \subset(0, \infty)$ of length $q$ contains at least one number of the form

$$
\varepsilon_{1} q+\varepsilon_{3} q^{3}+\varepsilon_{5} q^{5}+\ldots+\varepsilon_{2 n+1} q^{2 n+1}, \quad \varepsilon_{i} \in\{0,1\} .
$$

It follows that every interval $(x, x+2 \delta), x>a_{0}+q$, contains at least one $y_{k}$. Indeed, choose $b$ of the form (15) in $\left(x-a_{0}-q, x-a_{0}\right)$ and consider the numbers

$$
b+a_{0}<b+a_{1}<\ldots<b+a_{m} .
$$

It is clear that they all are in the sequence $\left(y_{k}\right)$. Since $b+a_{0}\left\langle x, b+a_{m}\right\rangle$ $b+a_{0}+q>x$ and since the difference of two consecutive elements is always less than $2 \delta$, it follows that at least one of them lies in $(x, x+\delta)$.

We have thus proved that $L(q) \leq 2 \delta$. Since $\delta>0$ was arbitrary, we conclude that $L(q)=0$.

The last part of the theorem follows from Lemma 8.

The following result completes Theorem 5:

Proposition 9. We have $L(\sqrt{2})=0$.

Proof. Fix $\delta>0$ and choose an integer $N>1 / \delta$. There exist two integers $0 \leq k<l \leq N$ such that the fractional part of $l \sqrt{2}-k \sqrt{2}$ is in $(0,1 / N)$. Taking integer multiples of $l \sqrt{2}-k \sqrt{2}$, it follows easily that there exists a finite sequence of integers $k_{1}<\ldots<k_{N}$ such that every interval of length $\delta$ contains at least one number having the same fractional part as one of $k_{i} \sqrt{2}, 1 \leq i \leq N$.

It follows that every interval $(x, x+\delta), x>k_{N} \sqrt{2}$, contains at least one $y_{k}$. Indeed, let $x<x^{\prime}<x+\delta$ and $1 \leq i \leq N$ be such that $x^{\prime}$ and $k_{i} \sqrt{2}$ have the same fractional part. Then $l:=x^{\prime}-k_{i} \sqrt{2}$ is a nonnegative integer and hence $x^{\prime}=l+k_{i} \sqrt{2}$ is in the sequence $\left(y_{k}\right)$.

Correction. We have proven in [3] that if $1<q<2$ and $L(q)=0$, then the number 1 has an infinite expansion containing arbitrarily long sequences of consecutive 0 digits (Theorem 4, part (c)). In the proof, at the bottom of page 388 , the sentence "It is equal to $y_{n}$ for some $n \geq 1$." should be changed to: "If $m$ is sufficiently large, then this number belongs to the interval $\left(y_{n-1}, y_{n}\right]$ for some $n \geq 2$." Next, the first sentence on the top of page 389 should be changed to: "It follows from (19) that $y_{n-1}<q^{m-k-n_{i_{k}}}$ and therefore $n_{1+i_{k}}>k+n_{i_{k}} \geq n_{i_{k}}$." The rest of the proof is the same. 
Let us note that the proof can easily be modified to prove, more generally, that under the same assumption $L(q)=0$, every $x \in(0,1 /(q-1))$ has an infinite expansion containing arbitrarily long sequences of consecutive 0 digits.

\section{Open problems}

1. Is it true that $l(q)>0$ if and only if $q$ is a Pisot number?

2. It would be interesting to determine the exact values of $l(q)$ and $L(q)$ for the Pisot numbers. Is it possible to adapt the proof of Proposition 3 for all Pisot numbers?

\section{References}

[1] Y. Bugeaud, On a property of Pisot numbers and related questions, Acta Math. Hungar. 73 (1996), 33-39.

[2] P. Erdős, I. Joó and M. Joó, On a problem of Tamás Varga, Bull. Soc. Math. France 120 (1992), 507-521.

[3] P. Erdős, I. Joó and V. Komornik, Characterization of the unique expansions $1=\sum q^{-n_{i}}$ and related problems, ibid. 118 (1990), 377-390.

Paul Erdős

Mathematical Institute of the Hungarian Academy of Sciences Reáltanoda u. 13-15 1053 Budapest, Hungary

István Joó

Department of Analysis Eötvös Loránd University Múzeum krt. 6-8 1088 Budapest, Hungary
Vilmos Komornik Institut de Recherche Mathématique Avancée Université Louis Pasteur et CNRS 7, rue René Descartes 67084 Strasbourg Cedex, France E-mail: komornik@math.u-strasbg.fr 